\title{
Acute HIV, Rhabdomyolysis, Renal failure, and Hepatitis: A CASE REPORT
}

Eve R. Merrill, $M D$

\section{Case Report}

A 19 year old African-American male with no significant past medical history presented to an outside hospital with two days of diffuse abdominal pain, myalgias, muscle weakness, and dark urine. Two weeks prior to presentation, the patient was treated as an outpatient with an unknown antibiotic for symptoms of a sore throat, nausea, and non-bloody, non-bilious emesis. The patient denied any sick contacts, recent travel, trauma, strenuous exercise, or the use of non-steroidal anti-inflammatory drugs, acetaminophen, alcohol, or illicit drugs. The patient reported being sexually active with one female partner and had no history of sexually transmitted infections. The patient was employed at a daycare center.

Vital signs and physical exam findings from the outside hospital were unavailable. Laboratory studies from the outside hospital showed a creatine phosphokinase (CPK) of 64,000 IU/L and a serum myoglobin of $37,036 \mathrm{ng} / \mathrm{ml}$, consistent with rhabdomyolysis. Urinalysis demonstrated $3+$ blood and 13 red blood cells. The patient was also in acute renal failure with a blood urea nitrogen of $44 \mathrm{mg} / \mathrm{dL}$ and creatinine of $6 \mathrm{mg} / \mathrm{dL}$. The patient was oliguric and uremic so hemodialysis was initiated at the outside hospital. The patient's labs were also significant for a transaminitis with an aspartate aminotransferase of 5,642 $\mathrm{U} / \mathrm{L}$ and an alanine aminotransferase of $543 \mathrm{U} / \mathrm{L}$. All other liver function tests were within normal limits. The patient had a negative urine drug screen and group A streptococcus rapid test. Viral testing for influenza A \& B, respiratory syncytial virus, Epstein-Barr virus (monospot), and HIV (ELISA) were all non-reactive. The patient was transferred to Thomas Jefferson University Hospital for a liver transplant evaluation and further management.

\section{Hospital Course}

Upon arrival to $\mathrm{TJUH}$, the patient was afebrile $\left(98.4^{\circ} \mathrm{F}\right)$, pulse of 99 beats per minute, blood pressure of 154/90 $\mathrm{mmHg}$, a respiratory rate of 24 breaths/min, and a pulse oximetry of $99 \%$ on room air. On review of systems, the patient complained of abdominal pain, myalgias, and fatigue. On physical exam, the patient appeared tired and ill. His abdomen was soft and mildly tender to palpation in all four quadrants. The patient had diffuse muscle tenderness but no joint effusions. His bilateral upper and lower extremities had non-pitting edema that extended to the elbows and knees. Upper extremity strength was $4 / 5$ bilaterally while lower extremity strength was $3 / 5$. The patient had no rashes, ulcers, asterixis, or jaundice. The rest of his physical exam was unremarkable. The labs on admission to TJUH showed a creatine phosphokinase of 234,417 IU/L, aspartate aminotransferase of 2,218 IU/L and alanine aminotransferase of $453 \mathrm{IU} / \mathrm{L}$. The blood urea nitrogen was $53 \mathrm{mg} / \mathrm{dL}$ and the creatinine was
$8 \mathrm{mg} / \mathrm{dL}$. The patient had a hemoglobin of $11.8 \mathrm{~g} / \mathrm{dL}$, a white blood cell count of $3.7 \mathrm{~B} / \mathrm{L}$ and platelets of $160 \mathrm{~B} / \mathrm{L}$. Urine studies demonstrated myoglobin, $3+$ protein, $3+$ blood, 158 red blood cells, no casts, and 3 white blood cells.

The patient continued to get daily dialysis while the primary team investigated the etiology of the patient's rhabdomyolysis, acute hepatitis, and acute renal failure. The patient's rhabdomyolysis and transaminitis improved throughout the hospitalization. Abdominal ultrasound of the liver showed no hepatomegaly but demonstrated prominent periportal walls, suggestive of inflammation. Since the patient's hepatic function was improving, the liver transplant team did not consider the patient a candidate for a liver transplant.

The patient's renal function was evaluated and renal ultrasound revealed mildly enlarged echogenic kidneys and no hydronephrosis. Renal biopsy was consistent with acute tubular necrosis and showed normal glomeruli, diffuse dilation of the proximal tubules, myoglobin containing casts, and granular eosinophilic casts. Although the patient was initially oliguric, his urine output improved during the hospitalization. He continued to require hemodialysis at discharge though the nephrologists were hopeful that this would only be temporary.

Autoimmune, rheumatologic, and infectious etiologies were further explored. Autoimmune labs for antinuclear antibody, antimitochondrial antibody, c-antineutrophil cytoplasmic antibody, p-antineutrophil cytoplasmic antibody, and anti-liverkidney microsome antibodies were all negative. Complement values were both within normal limits with a C3 of $81 \mathrm{mg} / \mathrm{dl}$ and a $\mathrm{C} 4$ of $15 \mathrm{mg} / \mathrm{dl}$. Hepatitis $\mathrm{A}, \mathrm{B}$, and $\mathrm{C}$ serologies, herpes simplex virus antibodies, rotavirus $\mathrm{Ag}$, and enterovirus stool RNA were all negative. The patient's throat, urine, blood, and stool cultures all showed no growth.

An HIV (ELISA) test was repeated to check for seroconversion. The HIV test was positive, the HIV RNA viral load was 273,000 copies/mL and the CD4 count was 290 cells $/ \mathrm{mm}^{3}$. The patient was diagnosed with an acute HIV infection that caused a viral myositis. The viral myositis stimulated rhabdomyolysis, which in turn produced acute tubular necrosis and transaminitis.

\section{Discussion}

Rhabdomyolysis is the breakdown of skeletal muscle and the release of the myocyte's intracellular components, including creatinine phosphokinase and myoglobin., ${ }^{1,2}$ Of note, myoglobinuria is not required for the diagnosis of rhabdomyolysis. ${ }^{2}$ Causes of rhabdomyolysis include trauma such as crush injuries, non-infectious sources (drugs, rheumatologic disorders, toxins) as well as infectious etiologies. ${ }^{2}$ Infectious sources include influenza, streptococcus, coxsackie virus and HIV. 
Patients infected with HIV have three stages in which rhabdomyolysis occurs. Most frequently, rhabdomyolysis is seen as a late manifestation of advanced AIDS when the HIV RNA stimulates lymphoid cells and inflammation around the myocytes. Second, rhabdomyolysis may also develop as a side effect of an antiretroviral medication during a chronic HIV infection. ${ }^{3}$ Typically, a primary HIV infection presents with mononucleosis or flu-like symptoms that include myalgias. ${ }^{4}$ In rare cases, rhabdomyolysis may result from acute HIV seroconversion. ${ }^{3,2}$ The exact mechanism in which acute HIV causes a viral myositis is not clear. Some possible explanations include the virus' direct invasion of the myocytes or viral-induced inflammation with the release of pro-inflammatory mediators. ${ }^{5}$

In order to make a definitive conclusion that an acute HIV infection induced rhabdomyolysis, a clinician must rule out other potential causes of rhabdomyolysis. For our patient, we ruled out many common viral and bacterial infectious causes as well as legal and illegal drugs, toxins, and rheumatologic disorders. Cytomegalovirus is a well-established cause of acute rhabdomyolysis. Medical literature contains very few case reports that can attribute acute HIV as the sole cause of rhabdomyolysis as the patient either had a co-infection with CMV or the patient was not tested for CMV., ${ }^{2,1}$ A literature review by McDonagh and Holman identify 23 case reports that attribute acute HIV as the etiology of rhabdomyolysis. However, when excluding cases in which the patient is co-infected with CMV or has another plausible explanation for rhabdomyolysis, McDonagh and Holman can only attribute four cases of rhabdomyolysis to acute HIV. ${ }^{2}$ Our patient tested positive for the CMV IgG $\mathrm{Ab}$ (9.72 IV) indicating a current or past CMV infection. Thus, we cannot exclude the possibility that CMV, and not HIV, was the infectious agent that produced the patient's rhabdomyolysis.

Although it may be rare, a patient who presents with acute rhabdomyolysis should be evaluated for a primary HIV infection, especially when another etiology cannot be identified. As in the case with our patient, an HIV test should be repeated as rhabdomyolysis may occur during the acute seroconversion period when the ELISA test may still be negative.

\section{References}

1. Delo D, Brett AS, Postic B. Primary HIV infection presenting with acute rhabdomyolysis. Am J Med Sci. 2006; 332(1):46-7.

2. McDonagh CA, Holman RP. Primary human immunodeficiency virus type 1 infection in a patient with acute rhabdomyolysis. South Med J. 2003; 96(10): 1027-30.

3. Maniar, AJ, Tolan Jr, RW. Rhabdomyolysis as the Presenting Manifestation of Acute Human Immunodeficiency Virus Infection in an Adolescent: Case Report and Literature Review. Infectious Diseases in Clinical Practice. 17(1): 12-16, January 2009.

4. Rastegar DA, Claiborne CV, Fleisher AS, Matsumoto AK. A patient with primary human immunodeficiency virus infection who presented with acute rhabdomyolysis. Clinical Infectious Diseases. 2001; 32(3):502-4.

5. Fodili F, van Bommel EF. Severe Rhabdomyolysis and acute renal failure following recent Coxsackie B virus infection. Neth J Med 2003; 61:177.

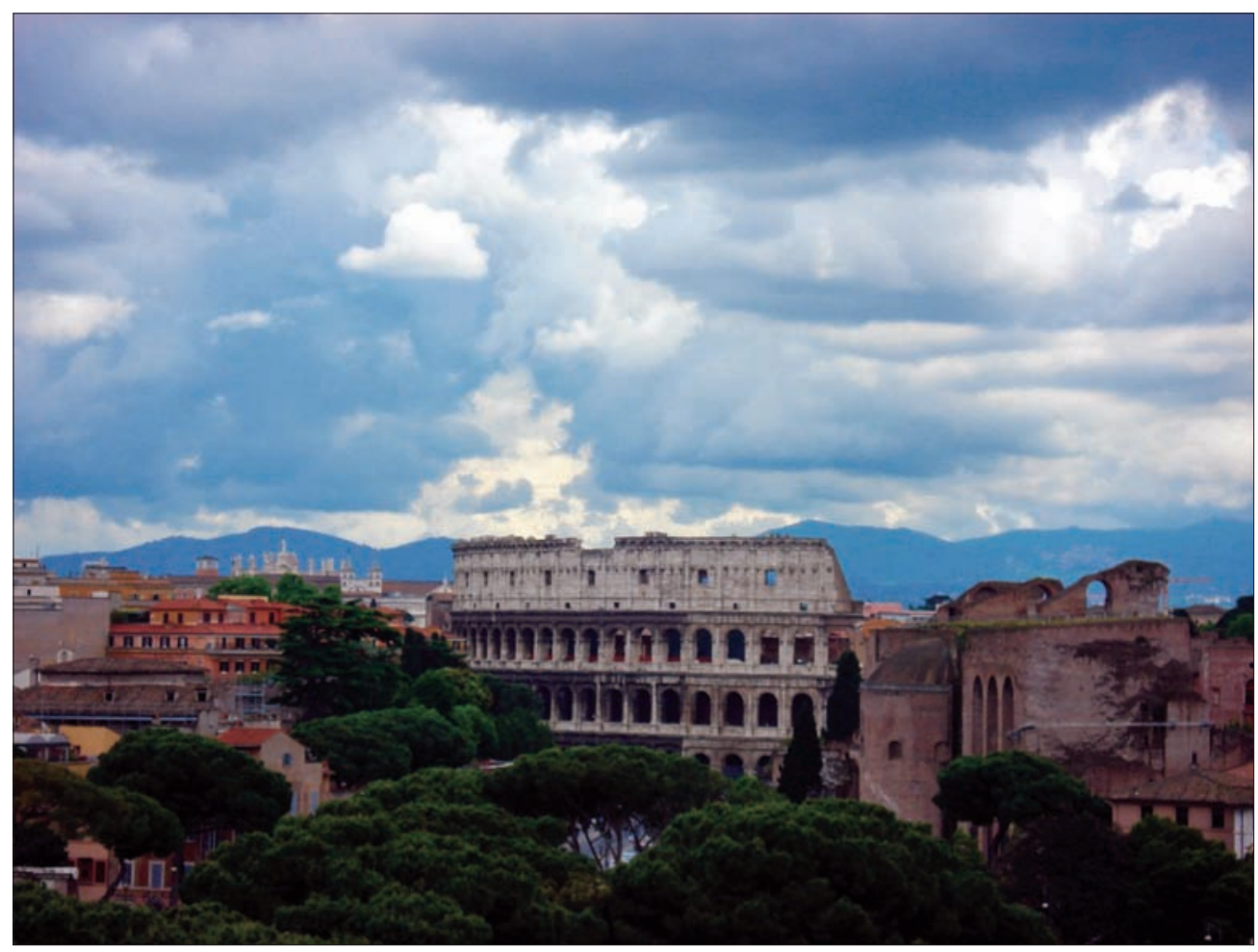

"Scenic Colosseum in the Distance", photograph by Paurush Shah, MD 